\title{
A EDUCAÇÃO AMBIENTAL NA GESTÃO DE RESÍDUOS DOS LABORATÓRIOS DA FEPAGRO/SEDE (FUNDAÇÃO ESTADUAL DE PESQUISA AGROPECUÁRIA)
}

\author{
Fernanda de Oliveira de Andrade Bertolo ${ }^{1}$, Adriana Ferreira Martins ${ }^{2}$, Dionísio Link ${ }^{3}$ \\ ${ }^{1}$ Bióloga, Mestre em Fitotecnia, Especialista em Educação Ambiental/UFSM - nanda_bio2@yahoo.com.br \\ ${ }^{2}$ Bióloga, Mestre em Ciência do Solo, Fundação de Pesquisa Agropecuária - biol.adriana@gmail.com \\ ${ }^{3}$ Eng. Agr., Dr. Dionísio Link, Professor Titular UFSM, Orientador - dionisiolink@yahoo.com.br
}

\section{RESUMO}

Educação Ambiental é um processo permanente no qual os indivíduos e a comunidade tomam consciência do seu meio ambiente e adquirem conhecimentos, valores, habilidades, experiências e determinação que os tornem aptos a agir e resolver problemas ambientais, presentes e futuros (UNESCO, 1987). Essa tem como objetivo sensibilizar e constitui uma forma abrangente de educação, que se propõe atingir todos os cidadãos, através de um processo pedagógico participativo permanente que procura estimular na pessoa uma consciência crítica sobre a problemática ambiental. A implementação de um programa de gestão de resíduos é algo que exige, antes de tudo, mudança de atitudes, e por isto, é uma atividade que traz resultado a médio e longo prazo, além de requerer realimentação contínua. Daí a importância do aspecto humano, pois o sucesso do programa está fortemente centrado na mudança de atitudes de todos os atores da unidade geradora. A divulgação é fundamental para a sensibilização e difusão de idéias e atitudes que o sustentarão (Jardim, 1998). Pensando nisto, se realizou um levantamento através de entrevista quantitativa dirigida aos integrantes dos laboratórios da FEPAGRO/Sede, localizada no município de Porto Alegre/RS. Os entrevistados de ambos os sexos apresentaram distribuição das faixas etárias entre 21 a 60 anos, no entanto o sexo masculino apresentou integrantes até próximo aos 70 anos. Dos cinco itens questionados aos entrevistados o que se refere a demanda de trabalho dos laboratórios, $70,59 \%$ assinalaram com ambas, tanto interna quanto externa e $23,53 \%$ apenas como interna. Quanto as análises realizadas pelos laboratórios $29,41 \%$ assinalaram Química e Física, 23,53\% Biológica, 17,65\% como Física e Biológica e 5,88\% tanto para todas e nenhuma das alternativas. Todos os entrevistados concordaram da produção de resíduos nos seus laboratórios de trabalho e, estes resíduos $94,12 \%$ são sólidos e líquidos. A classificação dos lixos produzidos foram agrupadas pelos entrevistados em: metais, vidros, plásticos, orgânicos e inorgânicos 35,30\%; vidros, plásticos, orgânicos e inorgânicos 23,53\%; 11,76\% tanto para vidros e inorgânicos como plásticos e orgânicos e 5,88\% para plásticos, orgânicos e inorgânicos, assim como para orgânicos e inorgânicos. Portanto, existe a importância da gestão de resíduos já que os laboratórios produzem análises e resíduos diversos para vários fins. Muito embora não haja uma legislação específica que trate do destino final de resíduos químicos oriundos das atividades de ensino e de pesquisa, isto não deve ser usado como um pretexto para a falta de gerenciamento destes rejeitos (Jardim, 2009).

Palavras chave: sensibilizar, processo pedagógico, rejeitos 
Monografias Ambientais ISSN: 2236-1308

\section{ABSTRACT}

Environmental Education is an ongoing process in which individuals and the community become aware of their environment, and acquires knowledge, values, skills, experience and $d$ determination to make it able to act and solve environmental problems, in the present and in the future (UNESCO, 1987). This have with objective raise awareness for one a comprehensive education, which proposes reach all citizens, through a continuous process and participatory teaching that seeks to stimulate the person in a critical awareness of the problem the environment. The implementation of a management program waste is something that requires, first of all changes attitudes, and therefore, is an activity that brings results the medium and long term, and requires feedback continuous. Hence the importance of human aspect because the success of the program is strongly focused on changing attitudes of all stakeholders of the generating unit. The disclosure is essential to raise awareness and spread of ideas and attitudes that sustain (Jardim, 1998). With this in mind, a survey was conducted through quantitative interview directed at members of FEPAGRO Labs / Headquarters, located in the municipality of Porto Alegre / RS. Respondents of both sexes present distribution of age groups between 21 and 60 years, however males presented members up close to 70 years. Of the five items asked respondents referred to the demand for laboratory work, noted with $70.59 \%$ both internally and externally, and only $23.53 \%$ as internal. The analysis performed by laboratories $29.41 \%$ indicated Chemistry and Physics, $23.53 \%$ Biological $17.65 \%$ as Physical and Biological and $5.88 \%$ for all and none of the alternatives. All respondents agreed that have production of waste in their laboratories work, and these residues are solid and net $94.12 \%$. The classification of waste was produced grouped by respondents on: metal, glass, plastics, organic and inorganic $35.30 \%$, glass, plastics, organic and inorganic $23.53 \%$, and $11.76 \%$ both for glass and plastics and inorganic and organic and $5.88 \%$ for plastics, organic and inorganic, as well as for organic and inorganic. Therefore, there is the importance of waste management since the laboratories produce and miscellaneous debris analysis for various purposes. Although there is no specific legislation that deals with the final disposal of waste chemicals from the teaching and research, this should not be used as an excuse for the lack of management of these wastes (Jardim, 2009).

Keywords: awareness, educational process, waste

\section{INTRODUÇÃO}

O gerenciamento dos resíduos gerados em laboratórios seja de unidades de ensino ou pesquisa é uma grande discussão, devido à problemática causada ao meio ambiente e a saúde pública. Isto se deve a diversidade de resíduos produzidos nos laboratórios, em função das amplas atividades desenvolvidas nos mesmos. Então, estes resíduos representam um problema de difícil gestão, porque não há uma única solução para extinguí-los por completo.

Sendo assim, várias ações devem ser realizadas simultaneamente, de modo a tornar a atividade gerenciadora possível e eficaz (Tavares \& Bendassolli, 2005). A geração de resíduos químicos em instituições de ensino e pesquisa no Brasil sempre foi um assunto muito pouco discutido. Sendo na maioria dos casos a gestão de resíduos gerados nas suas atividades rotineiras é inexistente, e devido à falta de um órgão fiscalizador, o descarte inadequado contínua a ser praticado (Jardim, 
1998). Essa falta de visão e o descarte inadequado levaram tanto Instituições de Pesquisas quanto Universidades a poluir o meio ambiente e promover o desperdício.

O gerenciamento de resíduos químicos em laboratórios de ensino e pesquisa no Brasil começou a ser amplamente discutido nos anos de 1990, sendo de vital importância para as grandes instituições geradoras (Afonso et al., 2003). Assim a implementação de um programa de gestão de resíduos é algo que exige, antes de tudo, mudança de atitudes, e por isto, é uma atividade que traz resultado a médio e longo prazo, além de requerer reeducação contínua. Daí a importância do aspecto humano, pois o sucesso do programa está fortemente centrado na mudança de atitudes de todos os atores da unidade geradora. A divulgação é fundamental para a sensibilização e difusão de idéias e atitudes que o sustentarão (Jardim, 1998).

Desde modo, de acordo com Jardim, 1998 as premissas (e condições) básicas para sustentar um programa de gestão de resíduos são quatro:

1. O apoio institucional irrestrito ao programa;

2. Priorizar o lado humano do programa frente ao tecnológico;

3. Divulgar as metas estipuladas dentro das várias fases do programa;

4. Reavaliar continuamente os resultados obtidos e as metas estipuladas.

É importante que a instituição esteja realmente disposta a implementar e sustentar um programa de gerenciamento de resíduos, pois o insucesso de uma primeira tentativa via de regra desacredita tentativas posteriores. Outro aspecto importante é o humano, pois o sucesso do programa está fortemente centrado na mudança de atitudes de todos os atores da unidade geradora. Este programa deve contemplar dois tipos de resíduos: o ativo (gerado continuamente nas atividades rotineiras na unidade geradora) e o passivo, que compreende todo aquele resíduo estocado, comumente não caracterizado, aguardando destinação final (o passivo inclui desde restos reacionais, passando por resíduos sólidos, até frascos de reagentes ainda lacrados mas sem rótulo). A caracterização desse passivo nem sempre é possível, embora algumas vezes seja possível chegar a seu conteúdo através de testes de identificação, contudo os laboratórios de pesquisa, onde os resíduos possuem uma maior diversidade quanto à natureza e à quantidade (Jardim, 1998; Afonso et al., 2003). É importante lembrar que esta caracterização prioriza o reciclo e o reuso de tudo que for possível, bem como habilita o resíduo para a sua destinação final (geralmente a incineração) (Jardim, 1998).

Ainda, dentro do passivo, é muito comum se encontrar frascos sem rótulos, mas que contêm reagentes caros, ainda íntegros, e cujo reuso depende apenas de testes analíticos relativamente simples. Neste caso convém devotar um tempo maior na caracterização destes resíduos. Cabe também lembrar que após a implementação do programa, com a rotulagem e identificação de todos os reagentes usados sendo feita em rotina, o passivo tende a ser cada vez menor e de mais fácil manejo (Jardim, 1998).

Segundo Jardim (1998), o ativo é aquele resíduo gerado rotineiramente nas atividades de ensino e de pesquisa, ou seja, o principal alvo de qualquer programa de gerenciamento. Independentemente de qual das atividades geradoras de resíduos (ensino ou pesquisa) serão abordadas, um programa de gerenciamento deve sempre adotar a regra da responsabilidade objetiva, ou seja, quem gerou o resíduos é responsável pelo mesmo, e praticar sempre a seguinte hierarquia das atividades:

1. Prevenção na geração de resíduos (perigosos ou não);

2. Minimizar a proporção de resíduos perigosos que são inevitavelmente gerados; 
3. Segregar e concentrar correntes de resíduos de modo a tornar viável e economicamente possível a atividade gerenciadora;

4. Reuso interno e externo;

5. Reciclar o componente material ou energético do resíduo;

6. Manter todo resíduo produzido na sua forma mais passível de tratamento;

7. Tratar e dispor o resíduo de maneira segura.

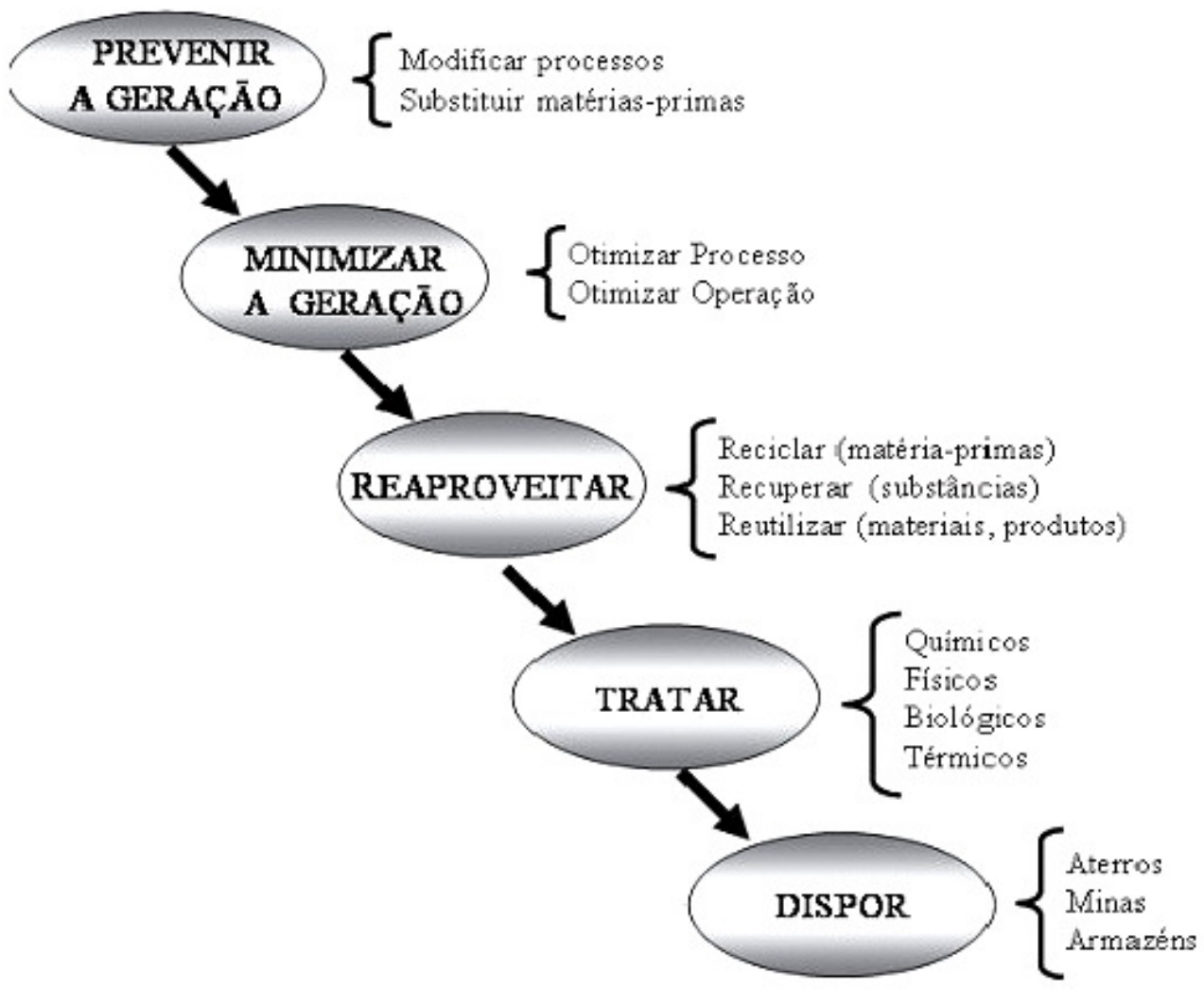

Figura 1 - Escala de prioridades a ser seguida quando da implantação de um PGR (programa de gestão de residuos) p. 733. (Tavares \& Bendassolli, 2005).

Estas atitudes podem ser facilmente traduzidas para a rotina de funcionamento da unidade geradora de várias maneiras. Sendo que a segregação dos resíduos em diferentes correntes tem como principal objetivo o de facilitar o seu tratamento e disposição final. $O$ reuso e o reciclo podem ser exercitados e fomentados dentro da unidade geradora. Entende-se por reuso o uso do resíduo como insumo, sem que o mesmo sofra qualquer pré-tratamento. Já o reciclo envolve o uso do material após algum tipo de tratamento. Cunha, 2001 comenta que os resíduos especiais aqueles que se encontram proibidos para o co-processamento tais como: agrotóxicos e alguns sais como, por exemplo, mercúrio e cádmio. Devem ter especial atenção quanto ao seu descarte para serem recuperadas e então enviadas para seu destino adequado. Com base nessas informações, o presente estudo teve como objetivo a análise quantitativa de questionários visando a implementação de um Programa de Gestão de Resíduos na Fundação Estadual de Pesquisa Agropecuária (FEPAGRO). 


\section{MATERIAL E MÉTODOS}

Tendo em vista a implementação de um PGR (Programa de Gestão de Resíduos) na FEPAGRO/Sede, localizada no município de Porto Alegre/RS. Foram realizados entrevistas com 17 pessoas, integrantes de laboratórios pertencentes a Fundação, sendo que a partir destas entrevistas - através de um questionário estruturado (Schwambach, 2007) contendo cinco itens com alternativas múltiplas, analisou-se quantitativamente tais dados obtidos.

\section{RESULTADOS E DISCUSSÃO}

Os integrantes dos laboratórios entrevistados apresentaram idades entre 21 e 60 anos de ambos os sexos, conforme Figura 1.

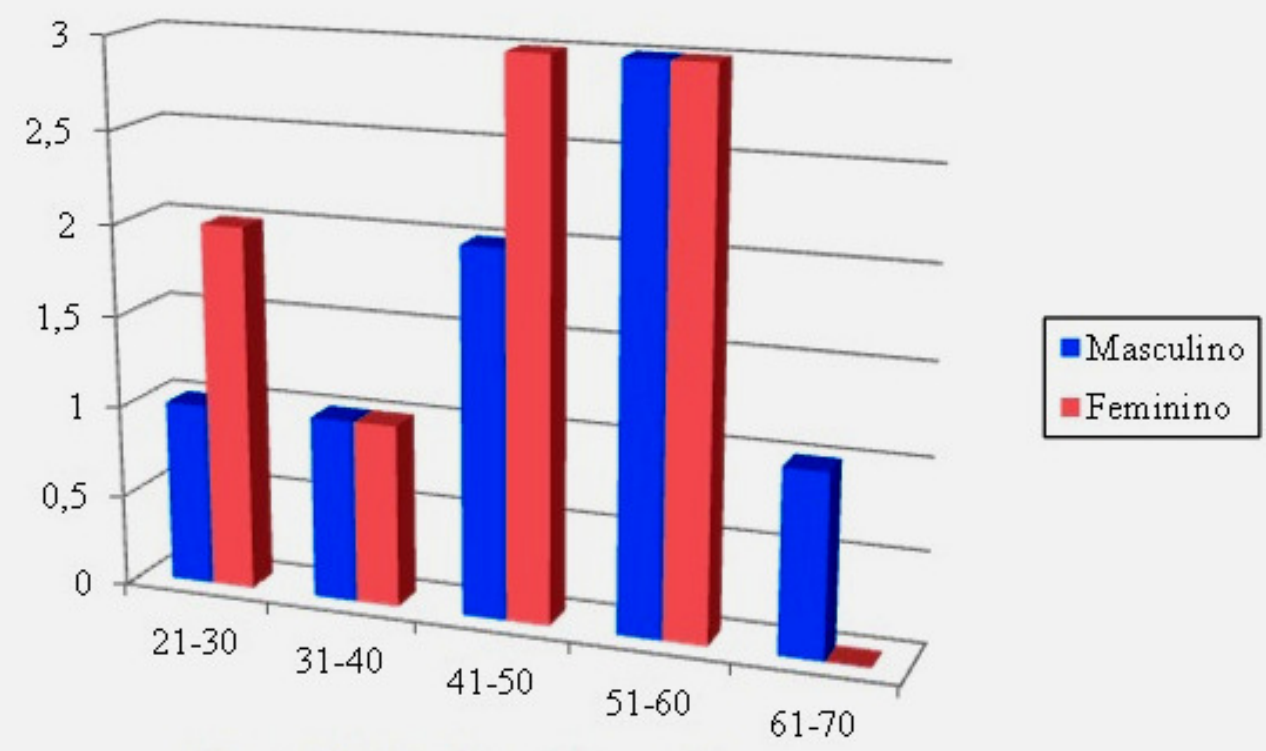

Figura 1 - Variação da faixa etária (em anos) e sexos.

Observa-se que há uma grande distribuição das faixas etárias atuantes dentro da Instituição, pois teve-se representantes a partir de 21 anos até próximo aos 70 anos, exceto no sexo feminino que apresentou representantes até a faixa que compreende 51-60 anos. Isto provavelmente se deve a esta Instituição ser de pesquisa, porque temos pesquisadores com uma grande experiência trabalhando em conjunto com os iniciantes, sendo isto de grande importância, pois o desenvolvimento de pesquisas não pode parar quando alguém se aposenta, por exemplo. Este sim deve ser contínuo e dinâmico.

Em relação ao item questionado referente a demanda de trabalho nos laboratórios (Figura 2), percebeu-se que a maior parte de trabalho desenvolvido nos mesmos, é a junção da interna e externa. Temos a demanda externa que também ocorre devido alguns destes laboratórios serem os primeiros a realizar análises deste tipo no Estado e por serem certificados e de referência no seu meio de trabalho. 


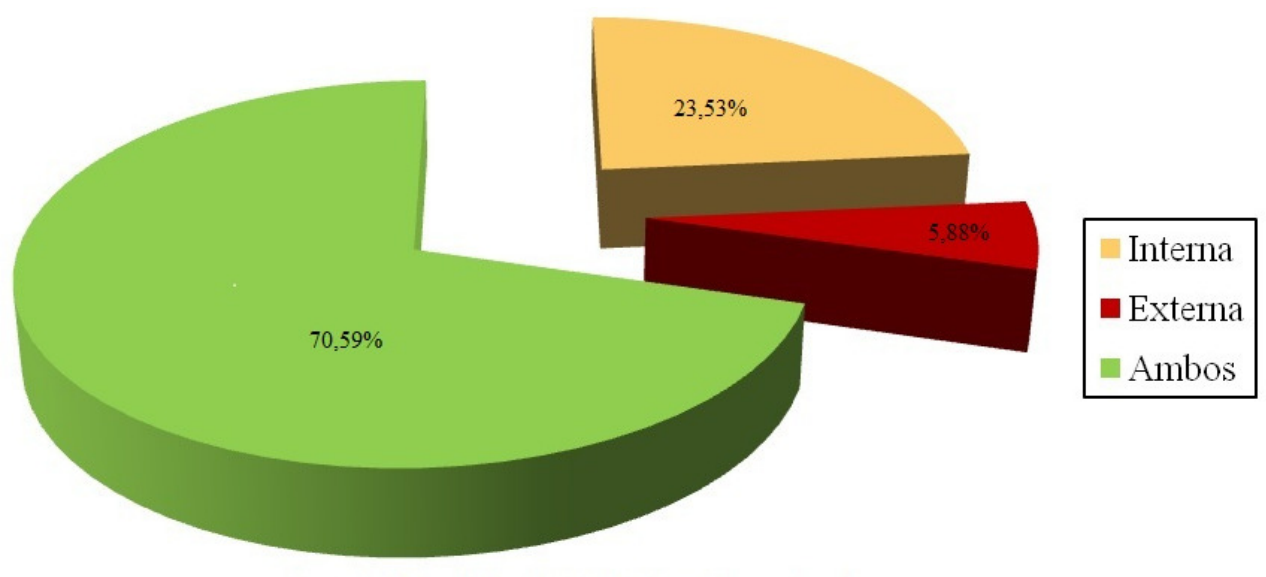

Figura 2 - Demanda de trabalho do laboratórios questionados

No questionamento que se refere quais análises os laboratórios realizam (Figura 3), demonstra que análises química e física são as mais realizadas $(29,41 \%)$, seguida de biológica $(23,53 \%)$ em conjunto representam mais da metade das análises dos laboratórios. Após temos física e biológica $(17,65 \%)$ e a menor se refere as alternativas todas e nenhuma, pois a Instituição possui laboratórios que apenas desenvolvem trabalhos e não análises.

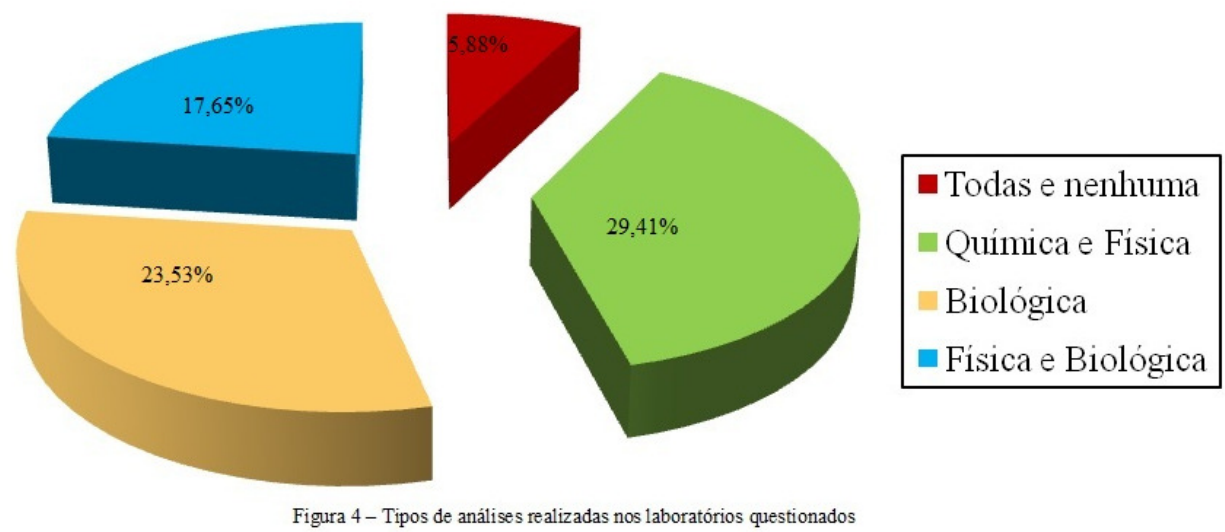

Quanto aos resíduos produzidos nos laboratórios, podemos observar na Figura 4 que todos os entrevistados concordaram da produção de resíduos nos seus laboratórios de trabalho e, estes resíduos $94,12 \%$ são sólidos e líquidos e, apenas 5,88\% produzem apenas líquidos. Isto se deve aos tipos de análises realizadas nos diferentes laboratórios. Por exemplo, ao se analisar uma bactéria, são utilizados diversos reagentes líquidos para fazer os meios de cultura tanto líquidos quanto sólidos para o seu crescimento, então assim são produzidos dois tipos de resíduos. Daí a explicação da junção dos dois no momento de resposta dos entrevistados. 


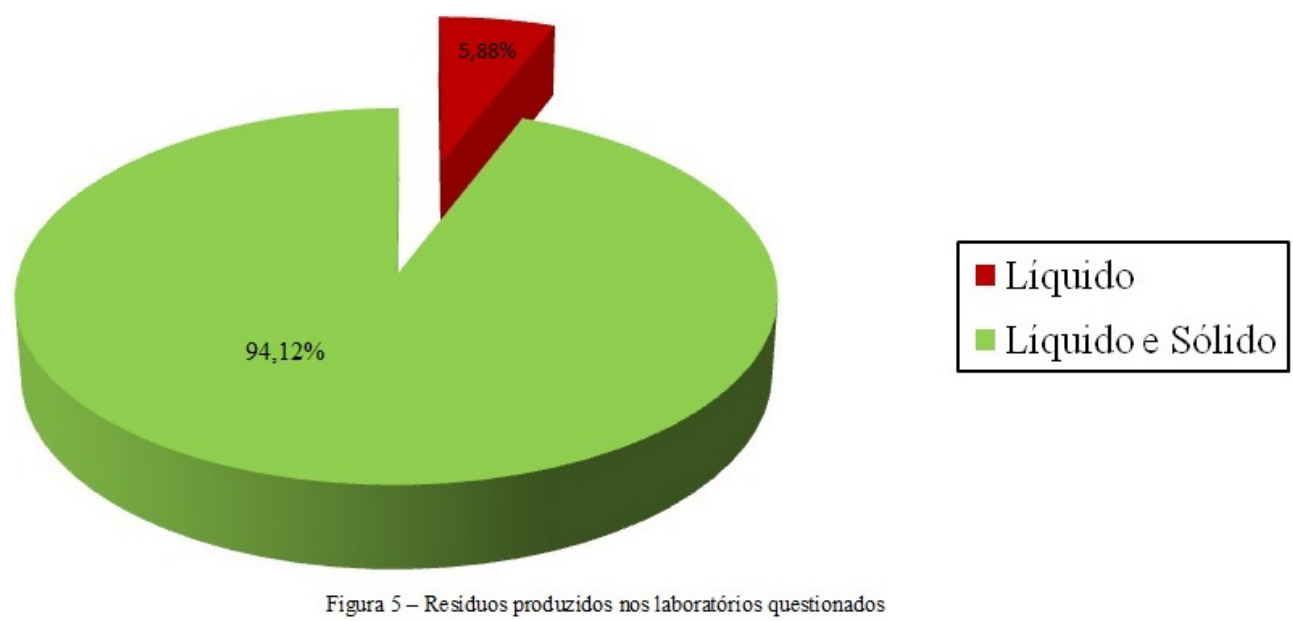

O lixo Orgânico são os restos de alimentos ou podas de árvores que podem ser transformado em adubo, através do processo de decomposição, e pode ser utilizado em hortas e jardins, servindo de nutrientes para plantas. Já o lixo Inorgânico é composto por materiais que após a coleta podem ser reciclados, bastando apenas sua separação em: metais, vidros, plásticos e papéis (Coleta Seletiva de Lixo CSB Prev, 2011). Portanto, nota-se que os entrevistados não perceberam que existem na verdade a separação em lixo orgânico e inorgânico e após, sim uma separação do inorgânico para sua posterior reciclagem ou reutilização. Conceito este também muitas vezes confundido pelas pessoas em geral.

Sendo a reciclagem um processo em que determinados tipos de materiais, cotidianamente reconhecidos como lixo, são reutilizados como matéria-prima para a fabricação de novos produtos. Além de se apresentarem com propriedades físicas diferentes, estes também possuem uma nova composição química, sendo este o fator principal que difere o reaproveitamento da reciclagem, conceitos estes muitas vezes confundidos. Esse processo é importante, nos dias de hoje, porque transforma aquilo que iria ou já se encontra no lixo em novos produtos, reduzindo resíduos que seriam lançados na natureza, ao mesmo tempo em que poupa matérias-primas, muitas vezes oriundas de recursos não renováveis e energia (Araguaia, 2011).

\section{Conclusões}

Existe a importância da gestão de resíduos já que os laboratórios produzem análises e resíduos diversos para vários fins. Muito embora não haja uma legislação específica que trate do destino final de resíduos químicos oriundos das atividades de ensino e de pesquisa, isto não deve ser usado como um pretexto para a falta de gerenciamento destes rejeitos (Jardim, 2009).

Portanto, o PGR na Instituição possui além da grande importância e necessidade, também um dos seus pré-requisitos principais que são a sensibilidade dos envolvidos neste contexto, ou seja, os integrantes/colaboradores dos laboratórios. Pois, sem o comprometimento destes não terão como serem implementadas as medidas necessárias para este programa.

\section{Referências}

AFONSO, J.C. et al., Gererenciamento de resíduos laboratoriais: recuperação de elementos e preparao para descarte final. Química Nova, v.26, n.4, São Paulo, 2003, p.602-611. 
CBS Previdência. COLETA SElETIVA DE LIXO. Disponível em: < http://www.cbsprev.com.br/web/images/Coleta Seletiva de lixo.pdf>. Acesso em: 07 nov. 2011.

CUNHA, C.J. O programa de gerenciamento dos resíduos laboratoriais do Depto de Química da UFPR. Química Nova, v.24, n. 3, São Paulo, 2001, p.424-427.

JARDIM, W.F. Gerenciamento de resíduos químicos em laboratórios de ensino e pesquisa. Química Nova, v.21, n.5, São Paulo, 1998, p.671-673.

- Gerenciamento de resíduos químicos. Disponível em: <http://lqa.iqm.unicamp.br/pdf/LivroCap11.PDF> Unicamp. Campinas, 2009.

SCHWAMBACH, K.H. Utilização de plantas medicinais e medicamentos no autocuidado no município de Teutônia, RS. Porto Alegre: UFRGS, 2007, 98p.

TAVARES, G.A.; BENDASSOLLI, J.A. Implantação de um programa de gerenciamento de resíduos químicos e águas servidas nos laboratórios de ensino e pesquisa no CENA/USP. Química Nova, v.28, n.4, São Paulo, 2005, p.732-738.

UNESCO. Internacional Strategy for Action in the field of Enviroment Education and Training for the 1990s. Disponível em: <http://unescodoc.unesco.org/images/0008/000805/080583eo.pdf>. Acesso: 07 nov. 2011. 